\title{
Modeling the Process-induced Dimensional Variations of General Curved Composite Components and Assemblies
}

\author{
Chensong Dong \\ Curtin University of Technology \\ GPO Box 1987, Perth, WA 6845, Australia
}

\begin{abstract}
Dimensional variations are induced during the processing of composite materials. General curved components are commonly used in composite structures. Their performance is affected by the dimensional variations associated with the manufacturing process. This paper presents a piecewise approach for predicting the dimensional variations of general curved composite components and assemblies. For a general curved composite component, it is first divided into a number of pieces of simple geometry. For each piece, the dimensional variation, i.e. spring-in, is calculated using the effective coefficients of thermal expansion. Based on the dimensional variation of each piece, the dimensional variations of the general curved component are calculated sequentially. This approach was validated against the finite element analysis. It shows that it offers excellent accuracy while avoiding time-consuming numerical computations. Besides general curved components, this approach can also be applied to composite assemblies. It provides the foundation for the tolerance analysis/synthesis of composites.
\end{abstract}

Keywords: Composites; general curved components; assemblies; dimensional variation; spring-in; effective coefficient of thermal expansion 


\section{Introduction}

The last four decades have seen a tremendous advancement in the science and technology of fiberreinforced composites. The low density, high strength, high stiffness to weight ratio, excellent durability, and design flexibility of composites are the primary reasons for their use in many structural components in aircraft, automotive, marine, and other industries [1]. Despite all these advantages, composite materials have not been as widely used as expected because of the complexity and cost of the manufacturing process. They are mainly used in the strength design such as aircraft, spacecraft and vehicles [2]. Poor dimensional control is one of the reasons causing these disadvantages. Dimensional variations lead to difficult assembly, costly rework, high waste rate, and structural failure, which limit the use of composites. Additional effort is needed to achieve better dimensional control. For example, NASA investigated the feasibility of SCRIMP to produce aircraft quality heavily-loaded primary structures. Additional technology development is required to achieve dimensional control and acceptable fiber volume fraction for thick structural elements [3].

Unlike processing of metals, composite processing is a net-shape process. The material and product form at the same time. Typical manufacturing processes are liquid composite molding processes, e.g. resin transfer molding and vacuum-assisted resin transfer molding processes. First, fiber preform is loaded into the mold cavity and the mold is closed. Second, resin is injected into the mold cavity by pressure or vacuum. Third, the filled mold is heated to some temperature often higher than the room temperature and resin is cured. Finally, the cured part is demolded from the mold. During the curing process, resin shrinks due to the chemical reaction (crosslinking). During the cooling-down process, the part is contracted. Due to the anisotropic nature of the material, dimensional variations are induced by coefficient of thermal expansion (CTE) mismatch and curing shrinkage of resin. 
"Spring-in" is the typical dimensional variations of angled parts, which is the decrease in the enclosed angle, as shown in Figure 1. Hahn and Pagano [4] performed an elastic analysis of the residual stresses in a thermoset matrix composite. Radford and Diefendorf [5] developed a simple mathematical formula to predict the spring-in of curved shaped parts, which was used by Huang and Yang [6] in their experimental studies. Kollar [7] presented an approximate analysis of springin. Jain and Mai $[8,9]$ developed a mechanics-based model using modified shell theory. Yoon and Kim [10] developed a computational method by applying the characterized properties to the classical lamination theory. All of these studies considered simple structures such as an L-shape. For predicting the spring-in of complex shapes, numerical simulation tools, e.g. finite element method or finite difference method, are often employed. Wang et al. [11] conducted a finite element analysis of spring-in using ABAQUS. Ding et al. [12] developed a 3-D finite element analysis procedure to predict spring-in resulting from anisotropy for both thin and thick angled composite shell structures.

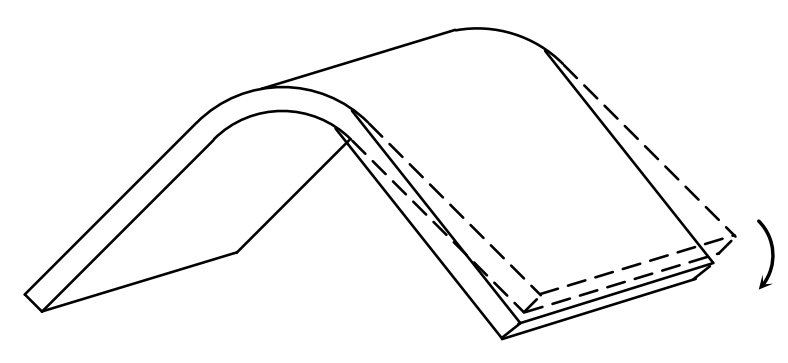

Figure 1: Spring-in

The mechanical behavior of composite materials is better represented by a viscoelastic model. Clifford et al. [13] developed a 3-D thermo-viscoelastic model by FEA to predict the residual stress and dimensional stability of large complex composite parts. Weitsman [14] developed a method for evaluating the residual stresses in cross-ply graphite-epoxy laminates which incorporated linear viscoelasticity throughout the cooldown stage. Wang et al. [15] studied residual stresses and 
warpage in multi-directional woven glass/epoxy laminates. The in-plane stresses and curvatures were calculated using a convolution integration form in the lamination theory expression. All the aforementioned studies neglected the stresses developed before cooldown. A few studies also addressed the residual stresses developed before cooldown, i.e. during the curing process. White and Hahn $[16,17]$ studied the residual stress development during the curing of thin laminates numerically and experimentally. A 2-D finite difference thermo-chemical model was developed. The cure-dependent material properties were incorporated into a generalized plane-strain finite element model to predict the residual stress development in a graphite-epoxy composite. Li et al. [18] used a plane-strain, linearly elastic finite element model with temperature-dependent matrix properties to analyze the evolution of residual stresses in graphite-PEEK composites during curing. Wiersma et al. [19] developed a thermo-elastic model and extended it into a thermo-viscoelastic model. A plane-strain finite element process model COMPRO was developed to simulate the spring-in and warpage in the autoclave process [20, 21]. Zhu et al. [22] developed a fully 3-D coupled thermo-chemo-viscoelastic finite element model to simulate the heat transfer, curing, and residual stress development during the manufacturing cycle of thermoset composite parts. Wisnom et al. [23] presented a shear-lag analysis for the spring-in of curved thermoset composites to study the influence of part thickness on spring-in.

From the literature, it can be seen that the process induced dimensional variations for composites such as spring-in were studied either analytically or numerically. The application of analytical models is limited to simple geometries such as an L-shape. For complex shapes such as general curved composite components, the analytical models for spring-in cannot be applied straightforwardly. In this study, a piece-wise approach for predicting the dimensional variations of general curved composite components and assemblies was developed. This approach was validated against the finite element analysis. 


\section{Spring-in Model Based on Effective Coefficients of Thermal Expansion}

\subsection{Spring-in model}

In the beginning of a typical liquid composite molding process, resin is fully uncured and behaves as viscous fluid. During the curing process, resin is heated up to a temperature usually above the glass transition temperature. A significant increase in modulus and a reduction in specific volume begin to occur. When the curing is complete, resin exhibits traditional viscoelastic behavior at elevated temperatures and approaches elastic behavior at lower temperatures. After demolding, the composite is usually post-cured at an elevated temperature above $T_{g}$. During this process, the stresses induced by curing can be significantly relaxed [24-26]. For some resin systems, such as epoxy, the curing strain during conversion can be neglected in the analysis of residual stress [27]. Thus, the residual stresses and dimensional variations are assumed to be mainly due to the mismatch of thermal contraction during the cooling-down process. Since the mechanical properties of resin are highly dependent on temperature, the mechanical properties and CTE of laminates are also dependent on temperature.

As shown in Figure 2Figure 2, the mechanism of spring-in is illustrated by using an L-shape structure. The enclosed angle is denoted as $\phi$ and the radius of the curved section is denoted as $r$. The original arc length is given by $s=r(180-\phi)$. 


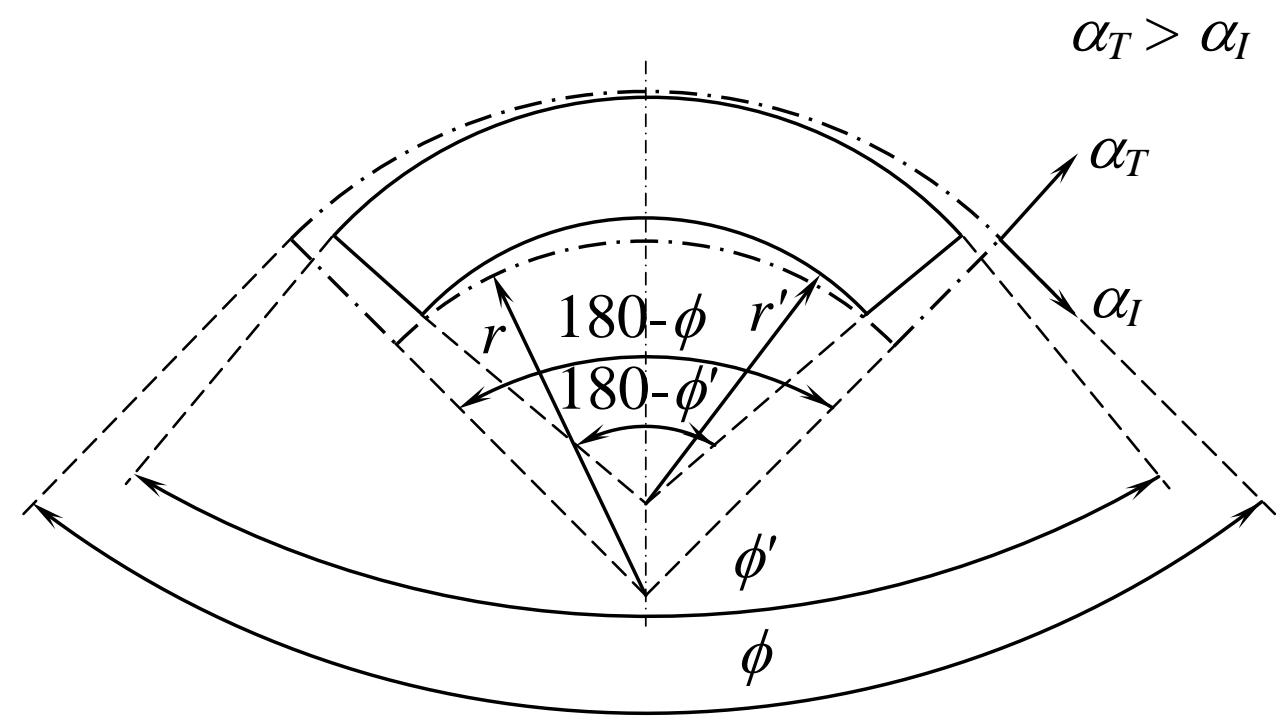

Figure 2: Mechanism of spring-in

When the part is cooled down from the curing temperature $T_{c}$ to the room temperature $T_{0}$, the radius and arc length after deformation are [6]

$$
\begin{aligned}
& r^{\prime}=r\left(1+\int_{T_{c}}^{T_{0}} \alpha_{T} d T\right) \\
& s^{\prime}=r\left(1+\int_{T_{c}}^{T_{0}} \alpha_{I} d T\right)(180-\phi)
\end{aligned}
$$

where $\alpha_{\mathrm{I}}$ and $\alpha_{T}$ are the in-plane and through-thickness CTE, respectively. The enclosed angle is given by

$$
\phi^{\prime}=180-\frac{s^{\prime}}{r^{\prime}}=180-\frac{1+\int_{T_{c}}^{T_{0}} \alpha_{I} d T}{1+\int_{T_{c}}^{T_{0}} \alpha_{T} d T}(180-\phi)
$$

Thus, the spring-in is given by 


$$
\Delta \phi=\frac{\int_{T_{c}}^{T_{0}}\left(\alpha_{T}-\alpha_{I}\right) d T}{1+\int_{T_{c}}^{T_{0}} \alpha_{T} d T}(180-\phi)
$$

Eqn. (3) shows that because of the mismatch of the in-plane CTE $\alpha_{I}$ and the through-thickness CTE $\alpha_{T}$, the enclosed angle decreases after the composite part is cured, which is commonly called “spring-in”.

\subsection{Derivation of effective coefficients of thermal expansion}

As shown in Figure 3Figure 3, in order to describe the elastic properties of a thin lamina, two righthanded coordinate systems, namely the 1-2-z system and the $x-y-z$ system, are defined. Both are in the plane of the lamina, and the $z$ axis is normal to this plane. In the 1-2-z system, axis 1 is along the fiber length and represents the longitudinal direction of the lamina, and axis 2 is normal to the fiber length and represents the transverse direction for the lamina. Together, they constitute the principal material directions in the plane of the lamina. In the $x-y$-z system, $x$ and $y$ axes represent the loading directions. Fibers are aligned at an angle $\theta$ with the positive $x$ direction [1]. 


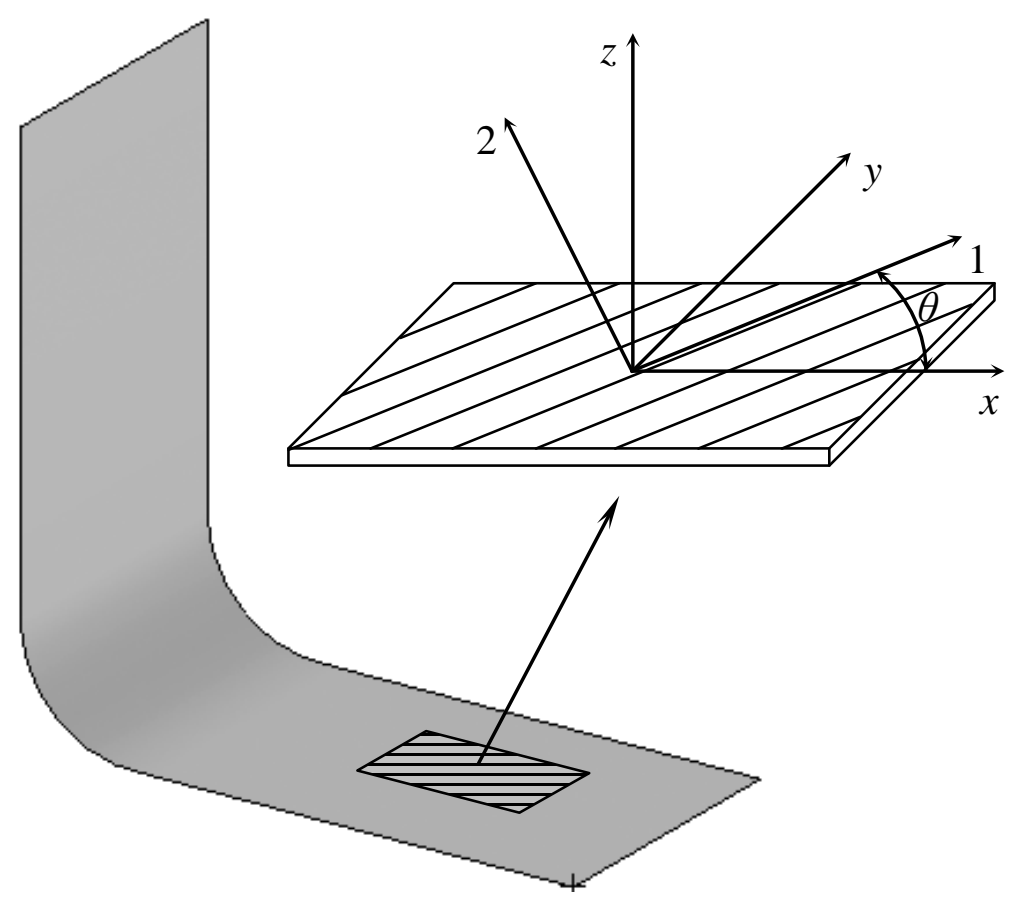

Figure 3: Definition of principal material axes and loading axes for a lamina

For a thin lamina in the 1-2-z coordinate system, its stiffness matrix can be expressed by $\mathbf{C}$. The elements of $\mathbf{C}$ are given in [28]. The stiffness matrix in the $x-y-z$ system is $\overline{\mathbf{C}}=\mathbf{T C} \mathbf{T}^{\mathbf{T}}$, where $\mathbf{T}$ is the transformation matrix.

The CTE in the $x-y-z$ system are related to the longitudinal CTE $\alpha_{11}$ and transverse CTE $\alpha_{22}$ as

$$
\begin{aligned}
& \alpha_{x x}=\alpha_{11} \cos ^{2} \theta+\alpha_{22} \sin ^{2} \theta \\
& \alpha_{y y}=\alpha_{11} \sin ^{2} \theta+\alpha_{22} \cos ^{2} \theta \\
& \alpha_{x y}=\left(\alpha_{11}-\alpha_{22}\right) \cos \theta \sin \theta \\
& \alpha_{z z}=\alpha_{33}=\alpha_{22}
\end{aligned}
$$

For a symmetric laminate, the constitutive relationship when only thermal stresses are considered is given by

$$
\left[\begin{array}{ccc}
\bar{C}_{11}^{e} & \bar{C}_{12}^{e} & \bar{C}_{13}^{e} \\
\bar{C}_{12}^{e} & \bar{C}_{22}^{e} & \bar{C}_{23}^{e} \\
\bar{C}_{13}^{e} & \bar{C}_{23}^{e} & \bar{C}_{33}^{e}
\end{array}\right]\left[\begin{array}{l}
\varepsilon_{x x} \\
\varepsilon_{y y} \\
\varepsilon_{z z}
\end{array}\right]=\left[\begin{array}{l}
T_{x x} \\
T_{y y} \\
T_{z z}
\end{array}\right] \Delta T
$$

where $\bar{C}_{i j}^{e}$ is the equivalent stiffness of the laminate [29], and $T_{x x}, T_{y y}$, and $T_{z z}$ are given as 


$$
\begin{aligned}
& T_{x x}=\sum_{i=1}^{n}\left(\bar{C}_{11}\right)_{i}\left(\alpha_{x x}\right)_{i}+\left(\bar{C}_{12}\right)_{i}\left(\alpha_{y y}\right)_{i}+\left(\bar{C}_{13}\right)_{i}\left(\alpha_{z z}\right)_{i}+\left(\bar{C}_{16}\right)_{i}\left(\alpha_{x y}\right)_{i} \\
& T_{y y}=\sum_{i=1}^{n}\left(\bar{C}_{12}\right)_{i}\left(\alpha_{x x}\right)_{i}+\left(\bar{C}_{22}\right)_{i}\left(\alpha_{y y}\right)_{i}+\left(\bar{C}_{23}\right)_{i}\left(\alpha_{z z}\right)_{i}+\left(\bar{C}_{26}\right)_{i}\left(\alpha_{x y}\right)_{i} \\
& T_{z z}=\sum_{i=1}^{n}\left(\bar{C}_{13}\right)_{i}\left(\alpha_{x x}\right)_{i}+\left(\bar{C}_{23}\right)_{i}\left(\alpha_{y y}\right)_{i}+\left(\bar{C}_{33}\right)_{i}\left(\alpha_{z z}\right)_{i}+\left(\bar{C}_{36}\right)_{i}\left(\alpha_{x y}\right)_{i}
\end{aligned}
$$

$\varepsilon_{x x}, \varepsilon_{y y}$, and $\varepsilon_{z z}$ can be solved from (6). The effective coefficients of thermal expansion were derived as

$$
\alpha_{x x}^{e}=\frac{\varepsilon_{x x}}{\Delta T} ; \alpha_{y y}^{e}=\frac{\varepsilon_{y y}}{\Delta T} ; \alpha_{z z}^{e}=\frac{\varepsilon_{z z}}{\Delta T}
$$

where $\alpha_{x x}$ and $\alpha_{y y}$ are the in-plane CTE and $\alpha_{z z}$ is the through-thickness CTE.

The effective coefficients of thermal expansion given by Eqn. (7) were validated against the finite element analysis using a representative volume element, since FEA is a well-accepted method to model composites on a layer-by-layer basis. An AS4 graphite/epoxy cross-ply laminate was assumed. The fiber volume fraction is $49 \%$ and the stacking sequence is $[0 / 90]_{8 s}$. A commercial FEA package MSC.Marc Mentat was employed in this study. The boundary conditions used in FEA were as follows: The model was restricted to move in the $i(i=x, y, z)$ direction along the plane $i=0$. Periodic boundary conditions were applied along the opposite planes, which were achieved by defining links in MSC.Marc Mentat. The model underwent a unit temperature drop. The resulting deformation is as shown in Figure 4Figure-4. The CTE of the composite in the direction $i(i=x, y, z)$ is given by

$$
\alpha_{i i}=\frac{\Delta l_{i}}{l_{i} \Delta T}
$$

where $\Delta l_{i}$ is the displacement in the i direction and $l_{i}$ is the original length. The effective CTE calculated by the current method and FEA are as shown in Table 1. Good agreement is found. 

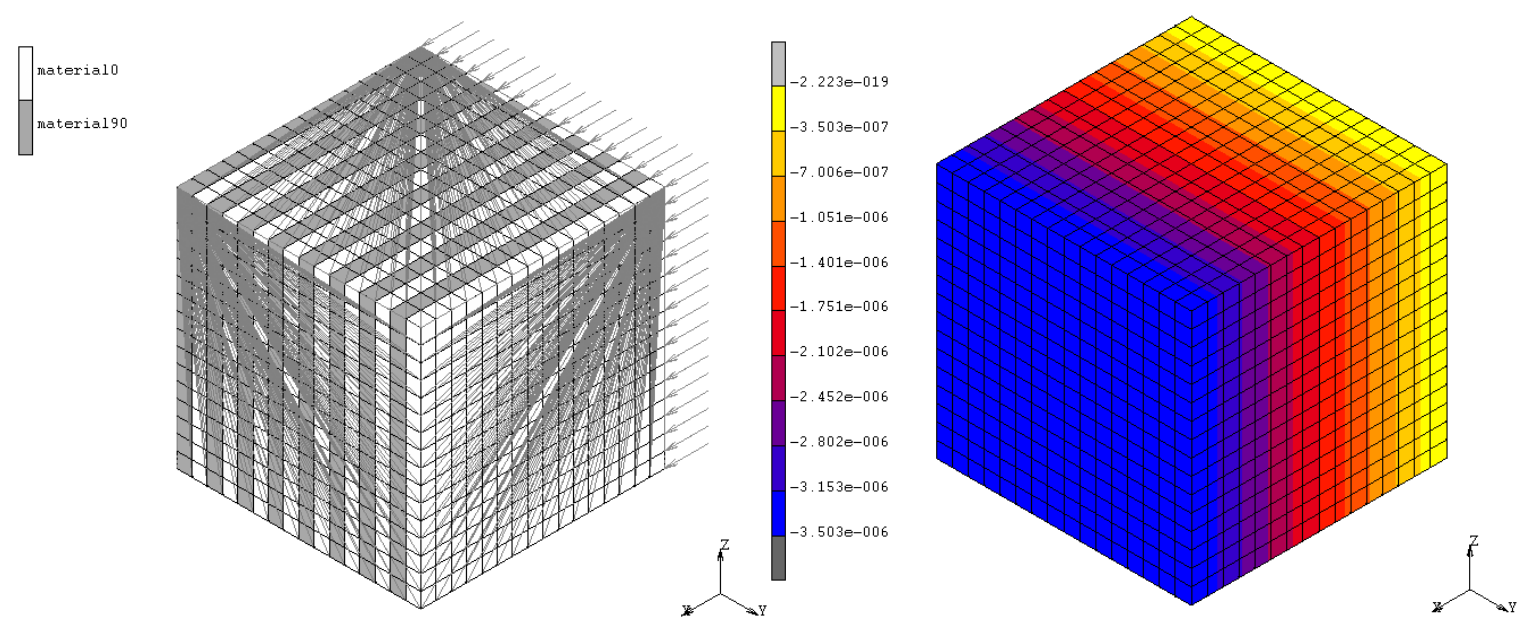

Figure 4: Prediction of effective CTE by FEA

Left: mesh; right: displacement. The contour shows the displacement in the $x$ direction in $\mathbf{m m}$.

Table 1: Effective CTE calculated by the current method and from FEA

\begin{tabular}{cccc}
\hline & $\alpha_{x x}\left(10^{-6} /{ }^{\circ} \mathrm{C}\right)$ & $\alpha_{y y}\left(10^{-6} /{ }^{\circ} \mathrm{C}\right)$ & $\alpha_{z z}\left(10^{-6} /{ }^{\circ} \mathrm{C}\right)$ \\
\hline Current method & 3.50 & 74.27 & 3.50 \\
FEA & 3.50 & 74.27 & 3.50 \\
\hline
\end{tabular}

\section{Dimensional Variations of General Curved Components}

For general curved composite components, the dimensional variations due to spring-in could not be calculated from Eqn. (4) directly. In this study, a piece-wise approach was developed to solve this problem. Because of the constant cross-section, this is a generalized plain strain problem. Thus, it can be solved in 2-D. This approach is illustrated by an example as shown in Figure 5.

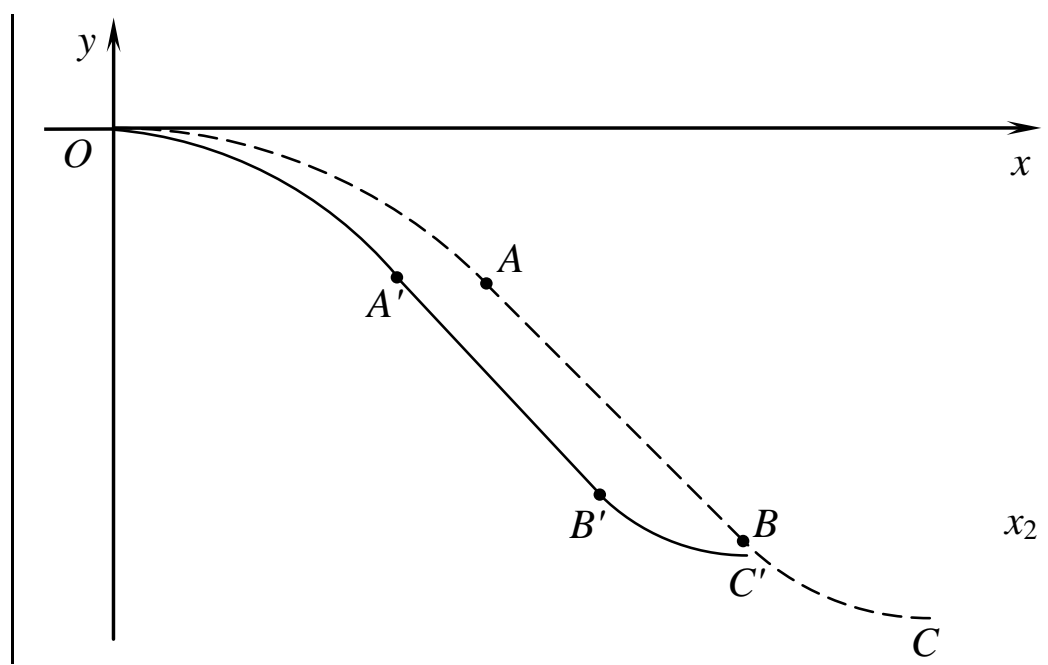


This general curve is decomposed into two arc sections $O A$ and $B C$ connected by a straight section $A B$. The global coordinate system is oxy. The coordinates of $A, B$, and $C$ are $\mathbf{A}=\left[x_{A}, y_{A}, 1\right]^{T}, \mathbf{B}=$ $\left[x_{B}, y_{B}, 1\right]^{T}$, and $C=\left[x_{C}, y_{C}, 1\right]^{T}$. The enclosed angle and radius are denoted as $\phi_{O A}$ and $r_{O A}$ for $O A$; and $\phi_{B C}$ and $r_{B C}$ for $B C$. Because of the process-induced shrinkage and spring-in, $A, B$, and $C$ move to $A^{\prime}, B^{\prime}$, and $C^{\prime}$.

For convenience, the concept of rotational angle is introduced. As shown in Figure 6, if the starting and end points of a general curve is denoted as $P_{i-1}$ and $P_{i}$, respectively, the rotational angle is given by

$$
\beta_{i}=\tau_{i}-\tau_{i-1}
$$

where $\tau_{i-1}=\arctan \left(\left.\frac{d y}{d x}\right|_{P_{i-1}}\right)$ and $\tau_{i}=\arctan \left(\left.\frac{d y}{d x}\right|_{P_{i}}\right)$.

The rotational angle can be positive or negative. As shown in Figure 6, if the spring-in causes counter-clockwise rotation, the rotational angle is defined as positive; if the spring-in causes clockwise rotation, the rotational angle is defined as negative. This is in accordance to the Cartesian rule. Eqn. (3) was modified using $\beta$ as

$$
\Delta \phi=-\frac{\int_{T_{c}}^{T_{0}}\left(\alpha_{T}-\alpha_{I}\right) d T}{1+\int_{T_{c}}^{T_{0}} \alpha_{T} d T} \beta
$$

Unlike Eqn. (3), where $\Delta \phi$ is always negative, $\Delta \phi$ in Eqn. (10) can be positive or negative. This is for the convenience of the derivation of the dimensional variations of the whole structure. 

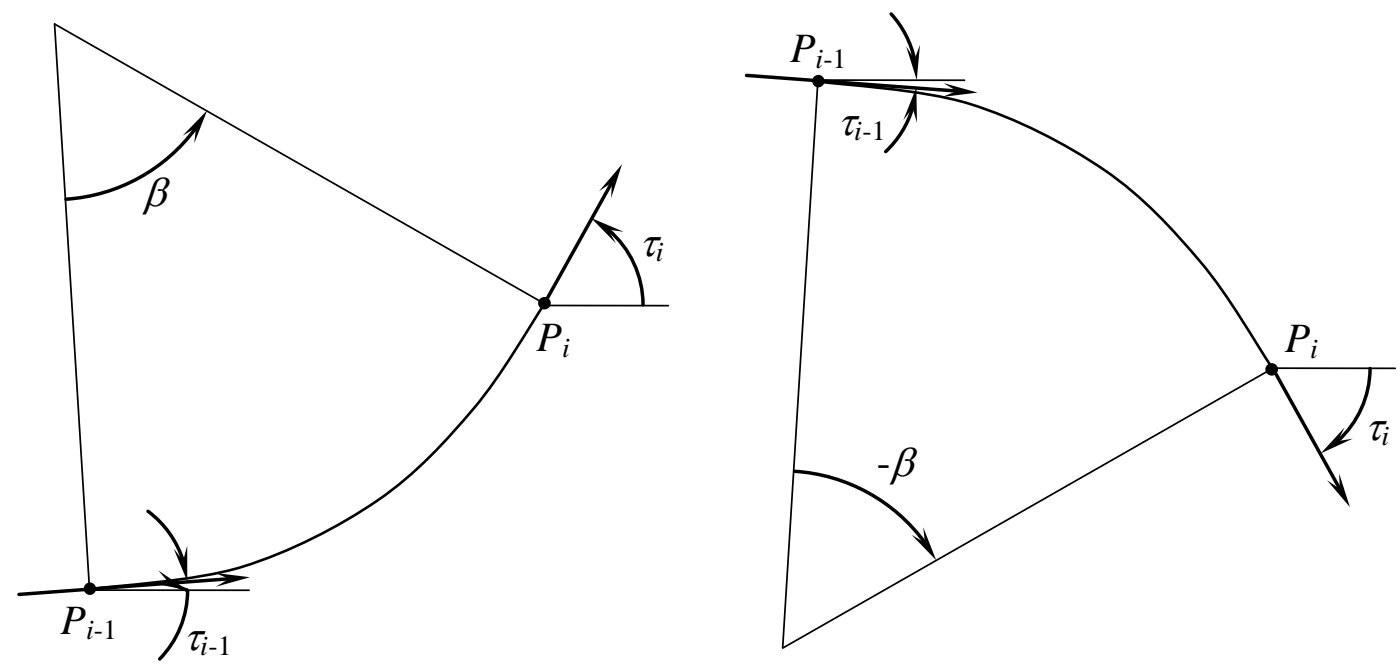

Figure 6: Definition of rotational angle (Left: positive; right: negative)

As shown in Figure 7, considering the arc section $O A$, a local coordinate system $o \xi_{1} \eta_{1}$ is constructed by rotating oxy clockwise. The angle $\chi_{O A}$ is defined as $\chi_{O A}=\arctan \left(\frac{y_{A}-y_{O}}{x_{A}-x_{O}}\right)$. The length of segment $O A$ is $|O A|=\sqrt{\left(x_{A}-x_{O}\right)^{2}+\left(y_{A}-y_{O}\right)^{2}}$. Thus, the radius of arc $O A$ is $r_{O A}=\frac{\sqrt{\left(x_{A}-x_{O}\right)^{2}+\left(y_{A}-y_{O}\right)^{2}}}{2\left|\sin \left(\frac{\beta_{O A}}{2}\right)\right|}$. Due to the through-thickness shrinkage, $r_{O A}$ decreases to $r_{O A}^{\prime}=\frac{\left(1+\alpha_{T} \Delta T\right) \sqrt{\left(x_{A}-x_{O}\right)^{2}+\left(y_{A}-y_{O}\right)^{2}}}{2\left|\sin \left(\frac{\beta_{O A}}{2}\right)\right|}$. Thus, in $o \xi_{1} \eta_{1}$, the coordinate of $A^{\prime}$ is

$$
\begin{gathered}
\xi_{A}=\frac{\left(1+\alpha_{T} \Delta T\right) \sqrt{\left(x_{A}-x_{O}\right)^{2}+\left(y_{A}-y_{O}\right)^{2}} \sin \left(\frac{\beta_{O A}-\Delta \phi_{O A}}{2}\right) \cos \left(\frac{\Delta \phi_{O A}}{2}\right)}{\sin \left(\frac{\beta_{O A}}{2}\right)} \\
\eta_{A}=\frac{\left(1+\alpha_{T} \Delta T\right) \sqrt{\left(x_{A}-x_{O}\right)^{2}+\left(y_{A}-y_{O}\right)^{2}} \sin \left(\frac{\beta_{O A}-\Delta \phi_{O A}}{2}\right) \sin \left(\frac{\Delta \phi_{O A}}{2}\right)}{\sin \left(\frac{\beta_{O A}}{2}\right)}
\end{gathered}
$$

The coordinate of $A^{\prime}$ in oxy can be derived by coordinate transformation as 


$$
\begin{aligned}
& x_{A}^{\prime}=\xi_{A} \cos \chi_{O A}-\eta_{A} \sin \chi_{O A} \\
& y_{A}^{\prime}=\xi_{A} \sin \chi_{O A}+\eta_{A} \cos \chi_{O A}
\end{aligned}
$$

Substituting Eqn. (11) into (12), the coordinate of $A^{\prime}$ in oxy is given by

$$
\begin{aligned}
& x_{A}^{\prime}=\frac{\left(1+\alpha_{T} \Delta T\right) \sqrt{\left(x_{A}-x_{O}\right)^{2}+\left(y_{A}-y_{O}\right)^{2}} \sin \left(\frac{\beta_{O A}-\Delta \phi_{O A}}{2}\right) \cos \left(\frac{\Delta \phi_{O A}}{2}+\chi_{O A}\right)}{\sin \left(\frac{\beta_{O A}}{2}\right)} \\
& y_{A}^{\prime}=\frac{\left(1+\alpha_{T} \Delta T\right) \sqrt{\left(x_{A}-x_{O}\right)^{2}+\left(y_{A}-y_{O}\right)^{2}} \sin \left(\frac{\beta_{O A}-\Delta \phi_{O A}}{2}\right) \sin \left(\frac{\Delta \phi_{O A}}{2}+\chi_{O A}\right)}{\sin \left(\frac{\beta_{O A}}{2}\right)}
\end{aligned}
$$

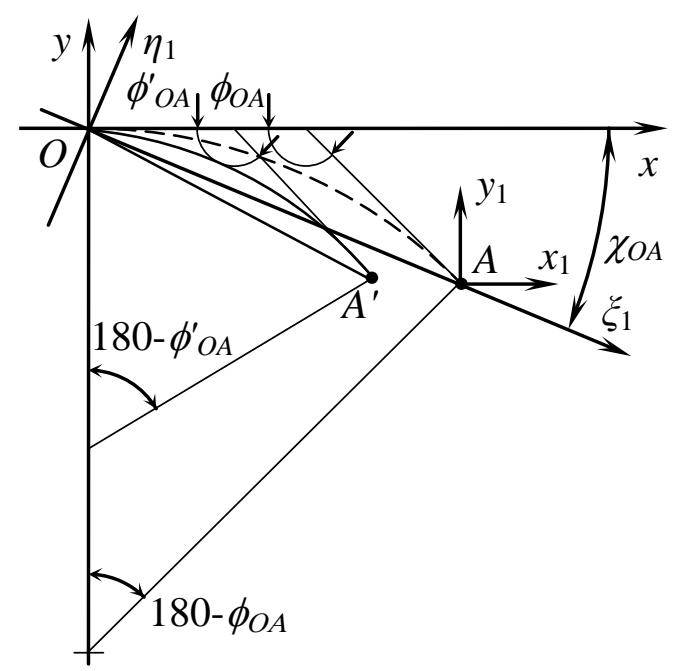

Figure 7: Dimensional variation of OA

As shown in Figure 8, considering the straight section $A B$ in $o x_{1} y_{1}$ where $A$ is the origin, there is no spring-in but only shrinkage induced. Without considering the rotation caused by $O A$, the coordinate of $B^{\prime}$ in $o x_{1} y_{1}$ is derived as

$$
\begin{aligned}
& x_{B A}^{\prime}=\left(1+\alpha_{I} \Delta T\right) x_{B A} \\
& y_{B A}^{\prime}=\left(1+\alpha_{I} \Delta T\right) y_{B A}
\end{aligned}
$$


Since the spring-in of $O A$ causes all the following pieces to rotate by $\Delta \phi_{O A}$, the coordinate of $B^{\prime}$ in oxy is

$$
\begin{aligned}
& x_{B}^{\prime}=x_{A}^{\prime}+x_{B A}^{\prime} \cos \Delta \phi_{O A}-y_{B A}^{\prime} \sin \Delta \phi_{O A} \\
& y_{B}^{\prime}=y_{A}^{\prime}+x_{B A}^{\prime} \sin \Delta \phi_{O A}+y_{B A}^{\prime} \cos \Delta \phi_{O A}
\end{aligned}
$$

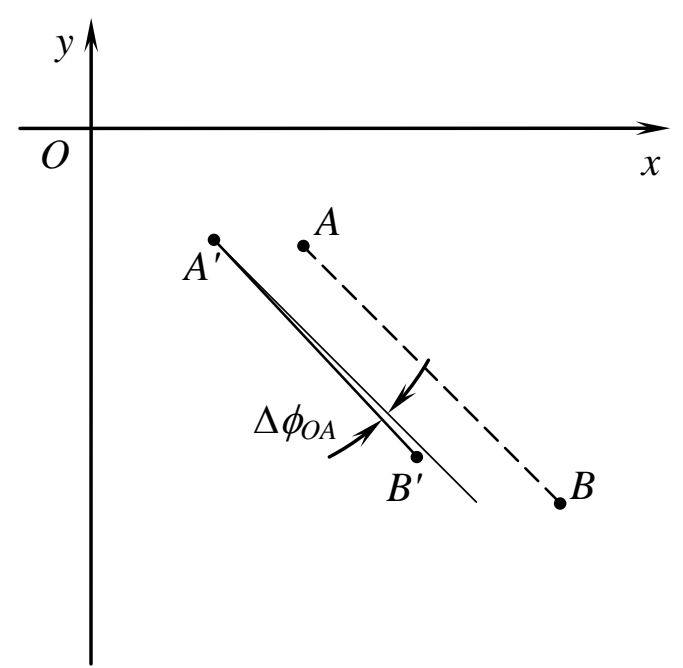

Figure 8: Dimensional variation of $A B$

As shown in Figure 9, a local coordinate system $o \xi_{2} \eta_{2}$ was constructed for arc BC. Likewise, the coordinate of $C^{\prime}$ in $o x_{2} y_{2}$ is derived as

$$
\begin{aligned}
& x_{C B}^{\prime}=\frac{\left(1+\alpha_{T} \Delta T\right) \sqrt{\left(x_{C}-x_{B}\right)^{2}+\left(y_{C}-y_{B}\right)^{2}} \sin \left(\frac{\beta_{B C}-\Delta \phi_{B C}}{2}\right) \cos \left(\frac{\Delta \phi_{B C}}{2}+\chi_{B C}\right)}{\sin \left(\frac{\beta_{B C}}{2}\right)} \\
& y_{C B}^{\prime}=\frac{\left(1+\alpha_{T} \Delta T\right) \sqrt{\left(x_{C}-x_{B}\right)^{2}+\left(y_{C}-y_{B}\right)^{2}} \sin \left(\frac{\beta_{B C}-\Delta \phi_{B C}}{2}\right) \sin \left(\frac{\Delta \phi_{B C}}{2}+\chi_{B C}\right)}{\sin \left(\frac{\beta_{B C}}{2}\right)}
\end{aligned}
$$

The coordinate of $C^{\prime}$ in oxy is

$$
\begin{aligned}
& x_{C}^{\prime}=x_{B}^{\prime}+x_{C B}^{\prime} \cos \left(\Delta \phi_{O A}+\Delta \phi_{A B}\right)-y_{C B}^{\prime} \sin \left(\Delta \phi_{O A}+\Delta \phi_{A B}\right) \\
& y_{C}^{\prime}=y_{B}^{\prime}+x_{C B}^{\prime} \sin \left(\Delta \phi_{O A}+\Delta \phi_{A B}\right)+y_{C B}^{\prime} \cos \left(\Delta \phi_{O A}+\Delta \phi_{A B}\right)
\end{aligned}
$$


i.e.

$$
\begin{aligned}
x_{C}^{\prime}= & x_{A}^{\prime}+x_{B A}^{\prime} \cos \Delta \phi_{O A}-y_{B A}^{\prime} \sin \Delta \phi_{O A} \\
& +x_{C B}^{\prime} \cos \left(\Delta \phi_{O A}+\Delta \phi_{A B}\right)-y_{C B}^{\prime} \sin \left(\Delta \phi_{O A}+\Delta \phi_{A B}\right) \\
y_{C}^{\prime} & =y_{A}^{\prime}+x_{B A}^{\prime} \sin \Delta \phi_{O A}+y_{B A}^{\prime} \cos \Delta \phi_{O A} \\
& +x_{C B}^{\prime} \sin \left(\Delta \phi_{O A}+\Delta \phi_{A B}\right)+y_{C B}^{\prime} \cos \left(\Delta \phi_{O A}+\Delta \phi_{A B}\right)
\end{aligned}
$$

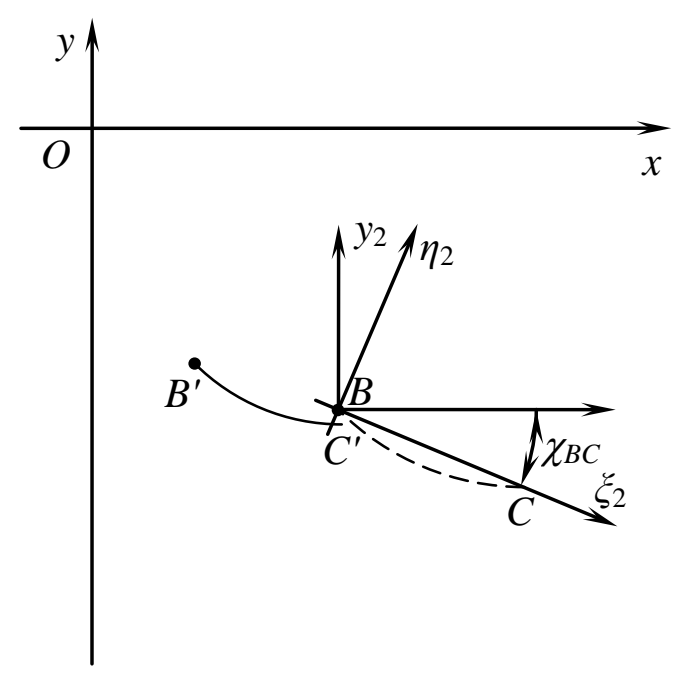

Figure 9: Dimensional variation of BC

In the vector form, Eqns. (13), (15), and (18) can be rewritten as

$$
\begin{aligned}
& \mathbf{A}^{\prime}=\mathbf{O A}^{\prime} \\
& \mathbf{B}^{\prime}=\mathbf{O} \mathbf{A}^{\prime}+\mathbf{R}_{\mathbf{z O A}} \cdot \mathbf{A}^{\prime} \mathbf{B}^{\prime} \\
& \mathbf{C}^{\prime}=\mathbf{O} \mathbf{A}^{\prime}+\mathbf{R}_{\mathrm{zOA}} \cdot \mathbf{A}^{\prime} \mathbf{B}^{\prime}+\mathbf{R}_{\mathrm{zOA}} \cdot \mathbf{R}_{\mathrm{zAB}} \cdot \mathbf{B}^{\prime} \mathbf{C}^{\prime}
\end{aligned}
$$

where $\mathbf{A}^{\prime}=\mathbf{O} \mathbf{A}^{\prime}=\left[\begin{array}{c}x_{A}^{\prime} \\ y_{A}^{\prime}\end{array}\right] ; \mathbf{B}^{\prime}=\left[\begin{array}{c}x_{B}^{\prime} \\ y_{B}^{\prime}\end{array}\right] ; \mathbf{C}^{\prime}=\left[\begin{array}{c}x_{C}^{\prime} \\ y_{C}^{\prime}\end{array}\right] ; \mathbf{R}_{\mathrm{zOA}}=\left[\begin{array}{cc}\cos \Delta \phi_{O A} & -\sin \Delta \phi_{O A} \\ \sin \Delta \phi_{O A} & \cos \Delta \phi_{O A}\end{array}\right]$;

$\mathbf{R}_{\mathbf{z A B}}=\left[\begin{array}{cc}\cos \Delta \phi_{A B} & -\sin \Delta \phi_{A B} \\ \sin \Delta \phi_{A B} & \cos \Delta \phi_{A B}\end{array}\right] ; \mathbf{A}^{\prime} \mathbf{B}^{\prime}=\left[\begin{array}{c}x_{B A}^{\prime} \\ y_{B A}^{\prime}\end{array}\right] ;$ and $\mathbf{B}^{\prime} \mathbf{C}^{\prime}=\left[\begin{array}{c}x_{C B}^{\prime} \\ y_{C B}^{\prime}\end{array}\right]$

For the convenience of practical applications, a sequence plot is constructed as shown in Figure 10.

The spring-in angles of each piece are marked on the sequence plot. For piece $i$, its spring-in angle $\Delta \phi_{i}$ will cause all the consequent pieces $i+1, i+2, \ldots, n$ to rotate by $\Delta \phi_{i}$. Figure 10 can be 
correlated with Eqn. (19). Based on the sequence plot, the dimensional variations of each node can be solved in a sequential way.

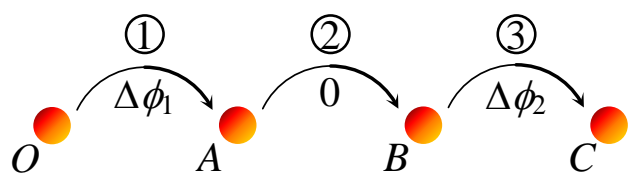

Figure 10: Sequence plot for the example part

The dimensional variations of this general curved component were calculated assuming AS4 fiber and epoxy resin. The material properties of AS4 fiber were taken from [30]. When the stacking sequence is $\left[0 / 90^{\circ}\right]_{6 s}$, the in-plane and through-thickness CTE were calculated as

$$
\begin{aligned}
& \alpha_{11}= \begin{cases}3.40 \times 10^{-6} /{ }^{\circ} \mathrm{C} & T=20^{\circ} \mathrm{C} \\
2.24 \times 10^{-6} /{ }^{\circ} \mathrm{C} & T=150^{\circ} \mathrm{C}\end{cases} \\
& \alpha_{22}= \begin{cases}72.85 \times 10^{-6} /{ }^{\circ} \mathrm{C} & T=20^{\circ} \mathrm{C} \\
71.39 \times 10^{-6} /{ }^{\circ} \mathrm{C} & T=150^{\circ} \mathrm{C}\end{cases}
\end{aligned}
$$

The rotational angles are $\beta_{O A}=45^{\circ}, \beta_{A B}=0$, and $\beta_{A B}=-45^{\circ}$. The spring-in angles were calculated as $\Delta \phi_{1}=-0.41^{\circ}, \Delta \phi_{2}=0$, and $\Delta \phi_{3}=0.41^{\circ}$. The dimensional variations of $A$ through $C$ calculated by the developed piece-wise approach are

$$
\Delta \mathbf{A}=\left[\begin{array}{l}
-0.16 \\
-0.23
\end{array}\right] \quad \Delta \mathbf{B}=\left[\begin{array}{l}
-0.54 \\
-0.57
\end{array}\right] \quad \Delta \mathbf{C}=\left[\begin{array}{l}
-0.62 \\
-0.68
\end{array}\right]
$$

where the unit is mm. For comparison, the dimensional variations of $A$ through $C$ from FEA are

$$
\Delta \mathbf{A}=\left[\begin{array}{l}
-0.17 \\
-0.23
\end{array}\right] \quad \Delta \mathbf{B}=\left[\begin{array}{l}
-0.54 \\
-0.57
\end{array}\right] \quad \Delta \mathbf{C}=\left[\begin{array}{l}
-0.63 \\
-0.68
\end{array}\right]
$$

It shows that good agreement exists between the developed piece-wise approach and FEA. 
By expanding this example, a general approach for the computation of dimensional variations of general curved components is derived. First, a Cartesian global coordinate system is defined, e.g. oxy in the example. Secondly, in oxy, the part can be divided into $n$ arc-like pieces with $n+1$ nodes $P_{0}, P_{1}, \ldots, P_{n}$. The spring-in and shrinkage of each piece can be calculated accordingly. A sequence plot for the general structure is as shown in Figure 11.
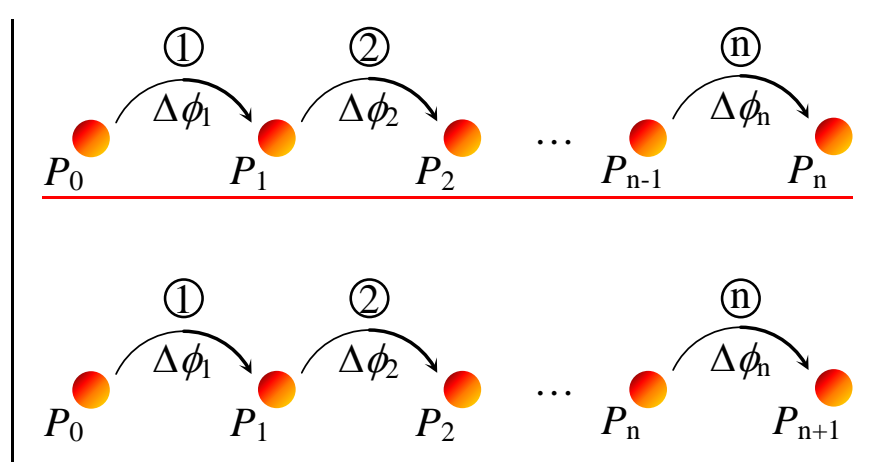

Figure 11: Sequence plot for a general part

By expanding Eqn. (19) and Figure 11, the deformed position of $P_{i}$ is given by

$$
\mathbf{P}_{\mathbf{n}}^{\prime}=\mathbf{P}_{\mathbf{0}}+\mathbf{P}_{\mathbf{0}}^{\prime} \mathbf{P}_{\mathbf{1}}^{\prime}+\sum_{i=2}^{n}\left[\left(\prod_{j=1}^{i-1} \mathbf{R}_{\mathbf{z j}}\right) \mathbf{P}_{\mathbf{i}-\mathbf{1}}^{\prime} \mathbf{P}_{\mathbf{i}}^{\prime}\right]
$$

where $\mathbf{P}_{\mathbf{i}-\mathbf{1}}^{\prime} \mathbf{P}_{\mathbf{i}}^{\prime}$ is the dimensional variation of $P_{i}$ in the local coordinate system with $P_{i-1}$ as the origin. $\mathbf{P}_{\mathbf{i}-\mathbf{1}}^{\prime} \mathbf{P}_{\mathbf{i}}^{\prime}$ can be found by

$$
\mathbf{P}_{\mathbf{i}-\mathbf{1}}^{\prime} \mathbf{P}_{\mathbf{i}}^{\prime}=\left[\begin{array}{c}
\frac{\left(1+\alpha_{T} \Delta T\right) \mathbf{P}_{\mathbf{i}-\mathbf{1}} \mathbf{P}_{\mathbf{i}} \mid \sin \left(\frac{\beta_{i}-\Delta \phi_{i}}{2}\right) \cos \left(\frac{\Delta \phi_{i}}{2}+\chi_{i}\right)}{\sin \left(\frac{\beta_{i}}{2}\right)} \\
\frac{\left(1+\alpha_{T} \Delta T\right) \mathbf{P}_{\mathbf{i}-\mathbf{1}} \mathbf{P}_{\mathbf{i}} \mid \sin \left(\frac{\beta_{i}-\Delta \phi_{i}}{2}\right) \sin \left(\frac{\Delta \phi_{i}}{2}+\chi_{i}\right)}{\sin \left(\frac{\beta_{i}}{2}\right)}
\end{array}\right]
$$

for arcs

$$
\mathbf{P}_{\mathbf{i}-\mathbf{1}}^{\prime} \mathbf{P}_{\mathbf{i}}^{\prime}=\left(1+\alpha_{I} \Delta T\right) \mathbf{P}_{\mathbf{i}-\mathbf{1}} \mathbf{P}_{\mathbf{i}}
$$

for straight lines 
The dimensional variation is given by

$$
\boldsymbol{\delta} \mathbf{P}_{\mathbf{n}}=\mathbf{P}_{\mathbf{0}}+\mathbf{P}_{\mathbf{0}}^{\prime} \mathbf{P}_{\mathbf{1}}^{\prime}+\sum_{i=2}^{n}\left[\left(\prod_{j=1}^{i-1} \mathbf{R}_{\mathbf{z j}}\right) \mathbf{P}_{\mathbf{i}-\mathbf{1}}^{\prime} \mathbf{P}_{\mathbf{i}}^{\prime}\right]-\mathbf{P}_{\mathbf{n}}
$$

In coordinate form, Eqn. (23) can be written as

$$
\begin{aligned}
& \delta x_{n}=x_{1}^{\prime}+\sum_{i=2}^{n}\left[x_{i, i-1}^{\prime} \cos \left(\sum_{j=1}^{i-1} \Delta \phi_{j}\right)-y_{i, i-1}^{\prime} \sin \left(\sum_{j=1}^{i-1} \Delta \phi_{j}\right)\right]-x_{n} \\
& \delta y_{n}=y_{1}^{\prime}+\sum_{i=2}^{n}\left[x_{i, i-1}^{\prime} \sin \left(\sum_{j=1}^{i-1} \Delta \phi_{j}\right)+y_{i, i-1}^{\prime} \cos \left(\sum_{j=1}^{i-1} \Delta \phi_{j}\right)\right]-y_{n}
\end{aligned}
$$

where

$$
\begin{aligned}
& x_{i, i-1}^{\prime}=\frac{\left(1+\alpha_{T} \Delta T\right) \sqrt{\left(x_{i}-x_{i-1}\right)^{2}+\left(y_{i}-y_{i-1}\right)^{2}} \sin \left(\frac{\beta_{i}-\Delta \phi_{i}}{2}\right) \cos \left(\chi_{i}+\frac{\Delta \phi_{i}}{2}\right)}{\sin \left(\frac{\beta_{i}}{2}\right)} \\
& y_{i, i-1}^{\prime}=\frac{\left(1+\alpha_{T} \Delta T\right) \sqrt{\left(x_{i}-x_{i-1}\right)^{2}+\left(y_{i}-y_{i-1}\right)^{2}} \sin \left(\frac{\beta_{i}-\Delta \phi_{i}}{2}\right) \sin \left(\chi_{i}+\frac{\Delta \phi_{i}}{2}\right)}{\sin \left(\frac{\beta_{i}}{2}\right)} \\
& x_{i, i-1}^{\prime}=\left(1+\alpha_{I} \Delta T\right) x_{i, i-1} \\
& y_{i, i-1}^{\prime}=\left(1+\alpha_{I} \Delta T\right) y_{i, i-1}
\end{aligned}
$$

\section{Conclusions}

A piece-wise approach for calculating the dimensional variations of general curved composite parts is presented in this paper. The effective coefficients of thermal expansion were derived and validated by FEA. The curved structure was divided into a number of pieces and the dimensional variations were calculated sequentially using the effective CTE. The approach was validated by FEA. It shows that the accuracy is satisfactory. Besides general curved components, this approach can be also applied to composite assemblies. This approach presented in this paper is potentially 
useful for the design of composite structures. Because of the simple and straightforward

mathematical form, the statistical distributions of the dimensional variations can be calculated

efficiently by incorporating the uncertainties of the spring in without going through many cycles of numerical computationsit is more efficient when the calculation of dimensional variations needs to be repeated. Thus, it provides the foundation for the tolerance analysis/synthesis of composite components and assemblies. For example, when the geometry of the component e.g. radius, enclosed angle, etc. is changed, a new geometric model and mesh need to be created when FEA is used, while only a few input numbers need to be changed when the approach presented in this paper is used. The savings in modeling and computation time are significant. Thus, it provides the foundation for the design optimization and tolerance analysis/synthesis of composite components and assemblies. The limitation of the current approach is that it is unable to calculate the dimensional variations of 3-D free-form surfaces, and this will be addressed in our future work.

\section{Acknowledgment}

The author is supported by the Curtin Research Fellowship.

\section{References}

1. Mallick, P. K. (1993) Fiber-Reinforced Composites: Materials, Manufacturing, and Design, New York, Marcel Dekker.

2. Lee, D. G., Lee, C. S., Lee, H. G., Hwang, H. Y. and Kim, J. W. (2004) Novel applications of composite structures to robots, machine tools and automobiles. Composite Structures, 2004(1-4): p. 17-39.

3. Shuart, M. J., Johnston, N. J., Dexter, H. B., Marchello, J. M. and Grenoble, R. W. (1998) Automated fabrication technologies for high performance polymer composites. NASA Langley Technical Report Server.

4. Hahn, H. T. and Pagano, N. J. (1975) Curing stress in composite laminates. Journal of Composite Materials, 9: p. 91-105.

5. Radford, D. W. and Diefendorf, R. J. (1993) Shape instabilities in composite resulting from laminate anistropy. Journal of Reinforced Plastics and Composites, 12(1): p. 58-75.

6. Huang, C. K. and Yang, S. Y. (1997) Warping in advanced composite tools with varying angles and radii. Composites Part A, 28(9-10): p. 891-893. 
7. Kollar, L. P. (1994) Approximate analysis of the temperature induced stresses and deformations of composite shells. Journal of Composite Materials, 28(5): p. 392-414.

8. Jain, L. K., Lutton, B. G., Mai, Y. W. and Paton, R. (1997) Stresses and deformations induced during manufacturing. Part II: a study of the spring-in phenomenon. Journal of Composite Materials, 31(7): p. 696-719.

9. Jain, L. K. and Mai, Y. W. (1997) Stresses and deformations induced during manufacturing. Part I: theoretical analysis of composite cylinders and shells. Journal of Composite Materials, 31(7): p. 673-695.

10. Yoon, K. J. and Kim, J.-S. (2001) Effect of thermal deformation and chemical shrinkage on the process induced distortion of carbon/epoxy curved laminates. Journal of Composite Materials, 35(3): p. 253-263.

11. Wang, J., Kelly, D. and Hillier, W. (2000) Finite element analysis of temperature induced stresses and deformations of polymer composite components. Journal of Composite Materials, 34(17): p. 1456-1471.

12. Ding, Y., Chiu, W. K. and Liu, X. L. (2001) Anisotropy related ‘spring-in’ of angled composite shells. Polymers \& Polymer Composites, 9(6): p. 393-401.

13. Clifford, S., Jansson, N., Yu, W., Michaud, V. and Manson, J.-A. (2006) Thermoviscoelastic anisotropic analysis of process induced residual stresses and dimensional stability in real polymer matrix composite components. Composites Part A, 37(4): p. 538-545.

14. Weitsman, Y. (1979) Residual thermal stresses due to cool-down of epoxy-resin composites. Journal of Applied Mechanics, 46(3): p. 563-567.

15. Wang, T. M., Daniel, I. M. and Gotro, J. T. (1992) Thermoviscoelastic analysis of residual stresses and warpage in composite laminates. Journal of Composite Materials, 26(6): p. 883-899.

16. White, S. R. and Hahn, H. T. (1992) Process modeling of composite materials: residual stress development during cure. Part I: model formulation. Journal of Composite Materials, 26(16): p. 2402-2422.

17. White, S. R. and Hahn, H. T. (1992) Process modeling of composite materials: residual stress development during cure. Part II: experimental validation. Journal of Composite Materials, 26(16): p. 2423-2454.

18. Li, M. C., Wu, J. J., Loos, A. C. and Morton, J. (1997) A plane-strain finite element model for process-induced residual stresses in a graphite/PEEK composite. Journal of Composite Materials, 31(3): p. 212-243.

19. Wiersma, H. W., Peeters, L. J. B. and Akkerman, R. (1998) Prediction of springforward in continuous-fiber/polymer L-shaped parts. Composites Part A, 29(11): p. 1333-1342.

20. Fernlund, G., Osooly, A., Poursartip, A., Vaziri, R., Courdji, R., Nelson, K., George, P., Hendrickson, L. and Griffith, J. (2003) Finite element based prediction of process-induced deformation of autoclaved composite structures using 2D process analysis and 3D structural analysis. Composites Structures, 62(2): p. 223-234.

21. Johnston, A., Vaziri, R. and Poursartip, A. (2001) A plane strain model for process-induced deformation of laminated composite structures. Journal of Composite Materials, 35(16): p. 1435-1469.

22. Zhu, Q., Geubelle, P. H., Li, M. and Tucker, C. L., III (2001) Dimensional accuracy of thermoset composites: simulation of process-induced residual stresses. Journal of Composite Materials, 35(2): p. 2171-2205.

23. Wisnom, M. R., Potter, K. D. and Ersoy, N. (2007) Shear-lag analysis of the effect of thickness on spring-in of curved composites. Journal of Composite Materials, 41(11): p. 1311-1324. 
24. Genidy, M. S., Madhukar, M. S. and Russell, J. D. (2000) A new method to reduce cureinduced stresses in thermoset polymer composites part II: closed loop feedback control system. Journal of Composite Materials, 34(22): p. 1905-1925.

25. Madhukar, M. S., Genidy, M. S. and Russell, J. D. (2000) A new method to reduce cureinduced stresses in thermoset polymer composites Part I: test method. Journal of Composite Materials, 34(22): p. 1882-1904.

26. Russell, J. D., Madhukar, M. S., Genidy, M. S. and Lee, A. Y. (2000) A new method to reduce cure-induced stresses in thermoset polymer composites part III: correlating stress history to viscosity degree of cure and cure shrinkage. Journal of Composite Materials, 34(22): p. 1926-1947.

27. Theriault, R. P. and Osswald, T. A. (1999) Processing induced residual stress in asymmetric laminate panels. Polymer Composites, 20(3): p. 493-509.

28. Chou, T.-W. (1992) Microstructural Design of Fiber Composites, Cambridge, U.K., Cambridge University Press.

29. Chen, H. J. and Tsai, S. W. (1996) Three-dimensional effective moduli of symmetric laminates. Journal of Composite Materials, 30(8): p. 906-917.

30. Adams, D. F. (1989) Properties characterization - mechanical/physical/hygrothermal properties test methods. IN LEE, S. M. (Ed.) Reference Book for Composites Technology Lancaster, PA, Technomic Pub Co. 